\title{
COMMISSION 33: STRUCTURE AND DYNAMICS OF THE GALACTIC SYSTEM
}

(STRUCTURE ET DYNAMIQUE DU SYSTEME GALACTIQUE)

PRESIDENT: K. Freeman

VICE-PRESIDENT: D. Spergel

ORGANIZING COMMITTEE: J. Binney, A. Fridman, O. Gerhard, J. Grindlay, A. Kalnajs, F. Matteucci, J. Palous, M. Perryman \& P. Whitelock

This report lists some of the major conferences that took place or were published during the current period in the areas of interest to Commission 33. It then presents in summary form some of the scientific highlights in these areas. An expanded version of this report, including more related conferences, full references and a report of activities from the former Soviet Union, appears on the mid-2000 WWW page of the Commission:

http://msowww.anu.edu.au/ kcf/kcf_ftp/IAU/comm33report_1999.html.

\section{WORKSHOPS, COLLOQUIA AND CONFERENCES}

In the period of this report, many workshops, colloquia and conferences were held on subjects of particular interest to Commission 33. The IAU sponsored meetings include:

Symposia:

179 NEW HORIZONS FROM MULTI-WAVELENGTH SKY SURVEYS, Baltimore, USA, August 26 - 30, 1996. Eds: B.J. McLean, D.A. Golombek, J.J.E. Hayes \& H.E. Payne

184 THE CENTRAL REGIONS OF THE GALAXY AND GALAXIES, Kyoto, Japan, August 18-22, 1997. Eds: Y. Sofue

187 COSMIC CHEMICAL EVOLUTION, Kyoto, Japan, August 26-30, 1997. Eds: J.W. Truran \& K. Nomoto

190 NEW VIEWS OF THE MAGELLANIC CLOUDS, Victoria, Canada, July 13-19, 1998. Eds. Y.-H. Chu, J. Hesser \& N. Suntzeff

Colloquia:

172 THE IMPACT OF MODERN DYNAMICS IN ASTRONOMY, Namur, Belgium, July 6-11, 1998. Eds. J. Henrard, S. Ferraz-Mello \& J.J. Binney

Non-IAU sponsored meetings:

THE GALACTIC HALO (The Third Stromlo Symposium), Canberra, Australia, August 17-21, 1998. Eds B. Gibson, T. Axelrod, \& M. Putman

GALAXY DYNAMICS, Rutgers University, August 8-12, 1998. Eds D. Merritt, M. Valluri \& J. Sellwood

GALACTIC HALOS, University of California, Santa Cruz, August 11-15, 1997. Ed D. Zaritsky

HIGH VELOCITY CLOUDS, Mt Stromlo Observatory, August 13-15, 1998. Eds B. Gibson \& M. Putman

GALAXY EVOLUTION: CONNECTING THE DISTANT UNIVERSE WITH THE LOCAL FOSSIL RECORD, Paris-Meudon Observatory, September 21-25, 1998.

STRUCTURE \& EVOLUTION OF STELLAR SYSTEMS, Petrozavodsk, Russia, August 13-17, 1995 (published 1997), Eds T. Agekian, A. Mullari \& V. Orlov 


\section{SCIENTIFIC HIGHLIGHTS}

Here is a brief overview of some scientific highlights in the area of interest of Commission 33 , for the period of this report. I am grateful to the members of the organising committee of the commission for their input to this section.

\subsection{Stellar Dynamics in Globular Cluster Cores}

Recent work on stellar interactions and populations in the cores of globular clusters has implications for the galactic system. The long-sought populations of cataclysmic variable (CV) binaries in globulars have been found from high resolution x-ray (ROSAT) and optical (HST) imaging and spectroscopy. A summary of the optical vs. $x$-ray properties of these systems is given by Grindlay (1999), which strongly suggest they may be dominated by magnetic CVs.

\subsection{Low Mass $\mathrm{X}$-ray Binaries and Neutron Stars in the Galaxy}

The high time resolution studies of low mass x-ray binaries (LMXBs) carried out with the Rossi X-ray Timing Explorer (RXTE) over the past 3 years have brought a series of breakthrough observations. Direct evidence of millisecond pulsations in LMXBs was finally found with the discovery of the millisecond x-ray pulsar SAX J1808.4-3658 (Wijnands and van der Klis 1998). The discovery of quasi-periodic oscillations (QPOs) at $\mathrm{kHz}$ frequencies and the relatively stable QPOs detected during $x$-ray bursts have all stimulated enormous theoretical and observational followup.

\subsection{Hipparchos}

The publication of the Hipparchos catalog by ESA in 1997 was a major milestone in observational galactic dynamics. The Hipparcos results are now being used in a wide variety of studies on the structure and dynamics of the Galaxy. Estimates of the local mass density in the disk (e.g. Crézé et al. 1998, Bienaymé 1999) give values of $\rho_{\odot} \simeq 0.09 M_{\odot} \mathrm{pc}^{-3}$. The authors conclude that there is no evidence for dark matter in the Galactic disk, and that any highly flattened component of dark matter is precluded. Dehnen \& Binney (1998) have studied the kinematics of main sequence stars as a function of colour, concluding that the Galactic potential is significantly non-axisymmetric at the solar radius, such that if spiral arms are responsible for the non-axisymmetry, they cannot be tightly wound.

Sellwood (1999) finds that the variations of the distribution function in the solar neighbourhood over small ranges in angular momentum, far less smooth than most theoretical work had previously supposed, support the picture of spiral patterns as dynamical instabilities driven by the substructure in the distribution function. Murali \& Dubinski (1999) are using the tidal streams from globular clusters to measure the mass distribution of the Milky Way, with the goal of determining the profile and shape of the inner halo.

The study of distance scales and their impact on Galactic structure and dynamics has been the subject of a number of papers, many of the topics being covered in Egret \& Heck (1999). In related work, Feast \& Whitelock (1997) have used the Hipparcos Cepheid motions to study the effects of Galactic rotation, deriving values of the Oort constants and the distance to the Galactic centre, while Olling \& Merrifield (1999) have used local stellar kinematics to derive mass models of the Galaxy from the local gradient of the rotation curve.

A number of studies have probed the membership and structure of clusters and moving groups: for example, de Zeeuw et al. (1999) have published an extensive study of nearby OB associations, linking their systematic patterns of proper motion to the Gould Belt system.

A concept and technology study was concluded by ESA in 1999 for a mission building on the Hipparcos principles, but extending the accuracies, number of objects and limiting magnitude by substantial factors. GAIA should be capable of measuring a billion or more objects to about $20 \mathrm{mag}$, providing accuracies at 15 mag of about 10 microarcsec, and hence direct distance determinations accurate to 10 per cent at distances of the Galactic 
centre. The satellite design includes comprehensive photometric classification, and radial velocities accurate to $1-5 \mathrm{~km} / \mathrm{s}$ for objects to about 16-17 mag. The technical studies have demonstrated that the mission is feasible for launch in 2009.

\subsection{Microlensing}

Over the past few years, observations of microlensing events have become an important tool for probing the mass distribution in our Galaxy. There have been over several hundred single lensing events and roughly one dozen binary lens events detected towards our galactic bulge (Alcock et al. 1999a; Udalski et al. 1997). The event rate towards the galactic center is roughly double the expected rate based on models for the galactic bar.

Observations of microlensing events towards the Large and Small Magellanic Clouds probe the composition of the galactic halo. Far more events have been detected towards the LMC and SMC than predicted. The nature and location of the lenses remain unknown. The lenses may be 0.5 solar mass white dwarfs or black holes in the galactic halo (Alcock et al. 1997) or stars within the Magellanic stream (Sahu 1994; Zhao 1998).

While observations of short duration single microlensing events cannot determine the location of the lenses, observations of long duration microlensing events or multiple microlensing events can determine or constrain the location of the lenses. Several of these more complex lensing events have been detected (Afonso 1999; Alcock et al. 1999b). In these cases, the lens events are most likely due to stars that lie within the Magellanic Clouds.

\subsection{Substructure in the Galactic Halo and Disk}

A proper motion study by Majewski et al. (1996) showed the presence of kinematic substructure in the galactic halo. Totten \& Irwin (1998) identified C-rich red giants in the halo which are presumed to have originated from disrupted dwarf galaxies. Martin \& Morrison (1998) analysed proper motions of RR Lyrae variables with results in general agreement with earlier work, but also isolating some interesting kinematic structure. Dehnen (1998) analysed the velocity distribution of nearby stars from Hipparcos data, identifying various moving groups. See also Chereul et al. (1999).

\subsection{The Inner Galaxy}

It is now well-established that the Galaxy contains a central bar-like bulge. In the past three years new photometric models based on the DIRBE NIR data have been published (eg Binney, Gerhard \& Spergel 1997). The bar is also seen in the apparent magnitude distributions of clump giant stars in different fields, from the distribution of IRAS variables and NIR starcounts. While the length of the bar is near 3-3.5 kpc and the bar angle $\phi$ with respect to the Sun-Center line is probably around $\phi=20-25^{\circ}$, there is still some debate about the axis ratios (within a range 10:[4-6]:[3-4]) and the detailed density structure. Dynamical models for the galactic bar were constructed with N-body simulation by Fux (1999).

Models for the gas flow in the potential of the Galactic bar and disk, by Englmaier \& Gerhard (1999), indicate that the corotation radius of the bar appears to be in the range [3-4.5] kpc, consistent with the length of the NIR bar. These models qualitatively reproduce a number of features in the $(1, v)$ diagrams such as the $3 \mathrm{kpc}$ arm. They are consistent with the observed spiral arm tangents and the inferred four-armed pattern for the Milky Way's spiral arms.

A further important conclusion from the NIR work is that the radial scale-length of the Galactic disk is short, $R_{D} \simeq 2.5 \mathrm{kpc}$. With its mass calibrated by the terminal velocity curve, the NIR disk has a local surface density $\Sigma_{\odot} \simeq 45 M_{\odot} p c^{-2}$ and accounts for most of the mass inside the solar circle (Gerhard 1999). While a dark halo is still required to explain a flat rotation curve at, and beyond, the solar circle, especially if $v_{\odot}>200 \mathrm{~km} \mathrm{~s}^{-1}$, the Milky Way disk is near-maximal, like those of other barred galaxies (e.g. Debattista \& Sellwood 1998). 


\subsection{The HI High Velocity Clouds}

From the kinematics of the high velocity clouds, there are growing indications that at least some of these clouds are Local Group objects, rather than clouds closely associated with the Galaxy (Blitz et al. 1999; Braun \& Burton 1998). HI observations by Putman et al. (1998) showed that the Magellanic Stream is indeed a tidal feature.

\subsection{The Dark Matter Content of the Galaxy}

Several estimates of the dark matter content of the Galaxy (e.g. Zaritsky 1999) indicate that the dark halo of the Galaxy extends out to a radius of about $200 \mathrm{kpc}$, and that its total mass is about $1 \times 10^{12} M_{\odot}$.

K. Freeman

President of the Commission

\section{References}

Afonso, C. et al. 1999, A\&A 344, L63.

Alcock, C. et al. 1997, ApJ, 486, 697.

Alcock, C. et al. 1999a, astro-ph/9907639

Alcock, C. et al. 1999b, ApJ, 518, 44.

Bienaymé, O. 1999, A\&A, 341, 86.

Binney, J. et al. 1997, MNRAS, 288, 365.

Blitz, L. et al. 1999, ApJ, 514, 818.

Braun R. \& Burton, W.B. 1998, A\&A, 341, 437.

Chereul, E. et al. 1999, A\&AS, 135, 5.

Crézé, M. et al. 1998, A\&A, 329, 920.

Debattista V., Sellwood J.A., 1998, ApJ, 439, L5.

Dehnen, W. 1998, AJ, 115, 2384.

Dehnen, W. \& Binney, J. 1998, MNRAS, 298, 387.

de Zeeuw, P.T. et al. 1999, AJ, 117, 354.

Egret,D. \& Heck, A. 1999, ASP Conf. Ser. 167.

Englmaier P., Gerhard O.E., 1999, MNRAS, 304, 512

Feast, M. \& Whitelock, P. 1997, MNRAS, 291, 683.

Fux R., 1999, A\&A, A\&A, 345, 787.

Gerhard O.E., 1999, in "Galaxy Dynamics", Eds Merritt D., Valluri, M., Sellwood, J.

Grindlay, J. 1999, ASP Conf. Ser. 157, 377.

Majewski, S., Munn, J., Hawley, S. 1996, ApJ 459, 73.

Martin, J. \& Morrison, H. 1998, AJ, 116, 1724.

Murali, C. \& Dubinski, J. 1999, AJ, 118, 911.

Olling, R. \& Merrifield, M. 1999, MNRAS, 297, 943.

Putman, M. et al. 1998. Nature, 394, 752.

Sahu, K.C. 1994, Nature, 370, 275.

Sellwood, J. 1999, ASP Conf. Ser. 160, 327.

Totten, E. \& Irwin, M. 1998, MNRAS 2941.

Udalski, A, Kubiak, M., \& Szymanski, M., 1997. Acta Astronomica, 47, 319

Wijnands, R. \& van der Klis, M. 1998, Nature, 394, 344.

Zaritsky, D. 1999. ASP Conf. Ser. 165, 34.

Zhao, H. 1998, MNRAS, 294, 139. 